\title{
Fixed point theory for cyclic generalized contractions in partial metric spaces
}

\author{
Ravi P Agarwal ${ }^{1,3}$, Maryam A Alghamdi ${ }^{2}$ and Naseer Shahzad ${ }^{3^{*}}$
}

\author{
* Correspondence: nshahzad@kau. \\ edu.sa \\ ${ }^{3}$ Department of Mathematics, King \\ Abdulaziz University, P.O. Box \\ 80203, Jeddah 21859, Saudi Arabia \\ Full list of author information is \\ available at the end of the article
}

\section{Abstract}

In this article, we give some fixed point theorems for mappings satisfying cyclical generalized contractive conditions in complete partial metric spaces.

\section{Introduction}

The well known Banach's fixed point theorem asserts that: If $(X, d)$ is a complete metric space and $f: X \rightarrow X$ is a mapping such that

$$
d(f(x), f(y)) \leq \lambda d(x, y)
$$

for all $x, y \in X$ and some $\lambda \in[0,1)$, then $f$ has a unique fixed point in $X$. Kannan [1] extended Banach's fixed point theorem to the class of maps $f: X \rightarrow X$ satisfying the following contractive condition:

$$
d(f(x), f(y)) \leq \lambda[d(x, f(x))+d(y, f(y))]
$$

for all $x, y \in X$ and some $\lambda \in(0,1 / 2)$. Reich [2] generalized both results using the contractive condition:

$$
d(f(x), f(y)) \leq \alpha d(x, y)+\beta d(x, f(x))+\gamma d(y, f(y))
$$

for each $x, y \in X$, where $\alpha, \beta, \gamma$ are nonnegative real numbers statisfying $\alpha+\beta+\gamma<$ 1.

Matkowski [3] used the following contractive condition:

$$
d(f(x), f(y)) \leq \varphi(d(x, y))
$$

for all $x, y \in X$, where $\phi: \mathbb{R}_{+} \rightarrow \mathbb{R}_{+}$is a nondecreasing function such that $\lim _{n \rightarrow \infty} \varphi^{n}(t)=0$ for all $t>0$.

In 1994, Matthews [4] introduced the notion of a partial metric space and obtained a generalization of Banach's fixed point theorem for partial metric spaces. Recently, Altun et al. [5] (see also Altun and Sadarangani [6]) gave some generalized versions of the fixed point theorem of Matthews [4]. Di Bari and Vetro [7] obtained some results concerning cyclic mappings in the framework of partial metric spaces. We recall below the definition of partial metric space and some of its properties (see $[4,5,8,9]$ ).

Definition 1 A partial metric on a nonempty set $X$ is a function $p: X \times X \rightarrow \mathbb{R}_{+}$ such that for all $x, y, z, \in X$ :

(c) 2012 Agarwal et al; licensee Springer. This is an Open Access article distributed under the terms of the Creative Commons Attribution License (http://creativecommons.org/licenses/by/2.0), which permits unrestricted use, distribution, and reproduction in any medium, provided the original work is properly cited. 


$$
\begin{aligned}
& \mathrm{p}_{1} x=y \Leftrightarrow p(x, x)=p(x, y)=p(y, y), \\
& \mathrm{p}_{2} p(x, x) \leq p(x, y) \\
& \mathrm{p}_{3} p(x, y)=p(y, x) \\
& \mathrm{p}_{4} p(x, y) \leq p(x, z)+p(z, y)-p(z, z)
\end{aligned}
$$

A partial metric space is a pair $(X, p)$ where $X$ is a nonempty set and $p$ is a partial metric on $X$. The function $p(x, y)=\max \{x, y\}$ for all $x, y \in \mathbb{R}_{+}$defines a partial metric on $\mathbb{R}_{+}$. Other interesting examples of partial metric spaces can be found in $[4,10,11]$. It is known [8] that each partial metric $p$ on $X$ generates a $T_{0}$ topology $\tau_{p}$ on $X$ which has as a base the family of open $p$-balls $\left\{B_{p}(x, \varepsilon): x \in X, \varepsilon>0\right\}$, where $B_{p}(x, \varepsilon)=\{y \in$ $X: p(x, y)<p(x, x)+\varepsilon\}$ for all $x \in X$ and $\varepsilon>0$.

If $p$ is a partial metric on $X$, then the function $p^{s}: X \times X \rightarrow \mathbb{R}_{+}$given by

$$
p^{s}(x, y)=2 p(x, y)-p(x, x)-p(y, y)
$$

defines a metric on $X$ (see [12]).

Let $(X, p)$ be a partial metric space.

A sequence $\left\{x_{n}\right\}$ in a partial metric space $(X, p)$ converges to a point $x \in X[4,5,8]$ if and only if $p(x, x)=\lim _{n \rightarrow \infty} p\left(x, x_{n}\right)$.

A sequence $\left\{x_{n}\right\}$ in a partial metric space $(X, p)$ is called a Cauchy sequence $[4,5,8]$ if there exists (and is finite) $\lim _{n, m \rightarrow \infty} p\left(x_{n}, x_{m}\right)$.

A partial metric space $(X, p)$ is said to be complete $[4,5,8]$ if every Cauchy sequence $\left\{x_{n}\right\}$ in $X$ converges, with respect to $\tau_{p}$, to a point $x \in X$ such that $p(x, x)=\lim _{n, m \rightarrow \infty} p\left(x_{n}, x_{m}\right)$.

It is evident that every closed subset of a complete partial metric space is complete.

Lemma $2[4,5,8]$ Let $(X, p)$ be a partial metric space.

$\left\{x_{n}\right\}$ is a Cauchy sequence in $(X, p)$ if and only if it is a Cauchy sequence in the metric space $\left(X, p^{s}\right)$.

(2)

A partial metric space $(X, p)$ is complete if and only if the metric space $\left(X, p^{s}\right)$ is complete. Furthermore, $\lim _{n \rightarrow \infty} p^{s}\left(x_{n}, x\right)=0$ if and only if

$$
p(x, x)=\lim _{n \rightarrow \infty} p\left(x_{n}, x\right)=\lim _{n, m \rightarrow \infty} p\left(x_{n}, x_{m}\right) .
$$

Definition 3 [13] Let $X$ be a nonempty set, $m$ a positive integer and $f: X \rightarrow X$ an operator. By definition, $X=\bigcup_{i=1}^{m} X_{i}$ is a cyclic representation of $X$ with respect to $f$ if

(i) $X_{i}, i=1, \ldots, m$ are nonempty sets;

(ii) $f\left(X_{1}\right) \subset X_{2}, \ldots, f\left(X_{m-1}\right) \subset X_{m}, f\left(X_{m}\right) \subset X_{1}$.

Definition 4 [13]A function $\phi: \mathbb{R}_{+} \rightarrow \mathbb{R}_{+}$is called a comparison function if it satisfies:

(i) $\phi$ is monotone increasing, i.e., $t_{1} \leq t_{2}$ implies $\phi\left(t_{1}\right) \leq \phi\left(t_{2}\right)$, for any $t_{1}, t_{2} \in \mathbb{R}_{+}$;

(ii) $\left(\phi^{n}(t)\right)_{n \in \mathbb{N}}$ converges to 0 as $n \rightarrow \infty$ for all $t \in \mathbb{R}_{+}$.

Definition 5 [13]A function $\phi: \mathbb{R}_{+} \rightarrow \mathbb{R}_{+}$is called a (c)-comparison function if it satisfies:

(i) $\phi$ is monotone increasing; 
(ii) there exist $k_{0} \in \mathbb{N}, a \in(0,1)$ and a convergent series of nonnegative terms $\sum_{k=1}^{\infty} v_{k}$ such that

$$
\varphi^{k+1}(t) \leq \alpha \varphi^{k}(t)+v_{k}
$$

for $k \geq k_{0}$ and any $t \in \mathbb{R}_{+}$.

Lemma 6 [13]If $\phi: \mathbb{R}_{+} \rightarrow \mathbb{R}_{+}$is a (c)-comparison function, then the following hold:

(i) $\phi$ is a comparison function;

(ii) $\phi(t)<t$, for any $t \in \mathbb{R}_{+}$;

(iii) $\phi$ is continuous at 0 ;

(iv) the series $\sum_{k=0}^{\infty} \varphi^{k}(t)$ converges for any $t \in \mathbb{R}_{+}$.

In [13], Păcurar and Rus discussed fixed point theorey for cyclic $\phi$-contractions in metric spaces and in [14], Karapinar obtained a fixed point theorem for cyclic weak $\phi$-contraction mappings still in metric spaces.

In this article, we prove some fixed point theorems for generalized contractions defined on cyclic representation in the setting of partial metric spaces.

\section{Main results}

Definition 7 Let $(X, p)$ be a partial metric space. A mapping $f: X \rightarrow X$ is called a $\phi$-contraction if there exists a comparison function $\phi: \mathbb{R}_{+} \rightarrow \mathbb{R}_{+}$such that

$$
p(f(x), f(y)) \leq \varphi(p(x, y))
$$

for all $x, y \in X$.

Definition 8 Let $(X, p)$ be a partial metric space, $m$ a positive integer, $A_{1}, \ldots, A_{m}$ nonempty closed subsets of $X$ and $Y=\bigcup_{i=1}^{m} A_{i}$. An operator $f: Y \rightarrow Y$ is called a cyclic $\phi$-contraction if

(i) $\bigcup_{i=1}^{m} A_{i}$ is a cyclic representation of $Y$ w.r.t $f$;

(ii) There exists a (c)-comparison function $\phi: \mathbb{R}_{+} \rightarrow \mathbb{R}_{+}$such that

$$
p(f(x), f(y)) \leq \varphi(p(x, y))
$$

for any $x \in A_{i v} y \in A_{i+1}$, where $A_{m+1}=A_{1}$.

Theorem 9 Let $(X, p)$ be a complete partial metric space, $m$ a positive integer, $A_{1}, \ldots$, $A_{m}$ closed nonempty subsets of $X, Y=\bigcup_{i=1}^{m} A_{i}, \varphi: \mathbb{R}_{+} \rightarrow \mathbb{R}_{+} a(c)$-comparison function and $f$ $: Y \rightarrow Y$ an operator. Assume that

(i) $\bigcup_{i=1}^{m} A_{i}$ is a cyclic representation of $Y$ w.r.t $f$;

(ii) $f$ is a cyclic $\phi$-contraction.

Then $f$ has a unique fixed point $x^{*} \in \bigcap_{i=1}^{m} A_{i}$ and the Picard iteration $\left\{x_{n}\right\}$ converges to $x^{*}$ for any initial point $x_{0} \in Y$.

Proof. Let $x_{0} \in Y=\bigcup_{i=1}^{m} A_{i}$, and set

$$
x_{n}=f\left(x_{n-1}\right), \quad n \geq 1 .
$$


For any $n \geq 0$ there is $i_{n} \in\{i, \ldots, m\}$ such that $x_{n} \in A_{i_{n}}$ and $x_{n+1} \in A_{i_{n+1}}$. Then by (2.1) we have

$$
p\left(x_{n}, x_{n+1}\right)=p\left(f\left(x_{n-1}\right), f\left(x_{n}\right)\right) \leq \varphi\left(p\left(x_{n-1}, x_{n}\right)\right) .
$$

Since $\phi$ is monotone increasing, we get by induction that

$$
p\left(x_{n}, x_{n+1}\right) \leq \varphi^{n}\left(p\left(x_{0}, x_{1}\right)\right) .
$$

By definition of $\phi$, thus letting $n \rightarrow \infty$ in (2.2), we obtain that

$$
\lim _{n \rightarrow \infty} p\left(x_{n}, x_{n+1}\right)=0 .
$$

On the other hand, since

$$
p\left(x_{n}, x_{n}\right) \leq p\left(x_{n}, x_{n+1}\right) \text { and } p\left(x_{n+1}, x_{n+1}\right) \leq p\left(x_{n}, x_{n+1}\right),
$$

then from (2.2) we have

$$
p\left(x_{n}, x_{n}\right) \leq \varphi^{n}\left(p\left(x_{0}, x_{1}\right)\right) \text { and } p\left(x_{n+1}, x_{n+1}\right) \leq \varphi^{n}\left(p\left(x_{0}, x_{1}\right)\right) \text {. }
$$

Thus, we have

$$
p^{s}\left(x_{n}, x_{n+1}\right) \leq 4 \varphi^{n}\left(p\left(x_{0}, x_{1}\right)\right) .
$$

Since $\phi$ is a $(c)$-comparison function, from Lemma 6, it follows that

$$
\lim _{n \rightarrow \infty} p^{s}\left(x_{n}, x_{n+1}\right)=0
$$

So for $k \geq 1$, we have

$$
\begin{aligned}
p^{s}\left(x_{n}, x_{n+k}\right) & \leq p^{s}\left(x_{n}, x_{n+1}\right)+\cdots+p^{s}\left(x_{n+k-1}, x_{n+k}\right) \\
& \leq 4 \sum_{m=n}^{n+k-1} \varphi^{m}\left(p\left(x_{0}, x_{1}\right)\right) .
\end{aligned}
$$

Again since $\phi$ is a (c)-comparison function, by Lemma 6, it follows that

$$
\sum_{m=0}^{\infty} \varphi^{m}\left(p\left(x_{0}, x_{1}\right)\right)<\infty .
$$

This implies that $\left\{x_{n}\right\}$ is a Cauchy sequence in the metric subspace $\left(Y, p^{s}\right)$. Since $Y$ is closed, the subspace $(Y, p)$ is complete. Then from Lemma 2, we have that $\left(Y, p^{s}\right)$ is complete. Let

$$
\lim _{n \rightarrow \infty} p^{s}\left(x_{n}, \gamma\right)=0
$$

Now Lemma 2 further implies that

$$
p(y, y)=\lim _{n \rightarrow \infty} p\left(x_{n}, y\right)=\lim _{n, m \rightarrow \infty} p\left(x_{n}, x_{m}\right) .
$$

Therefore, since $\left\{x_{n}\right\}$ is a Cauchy sequence in the metric space $\left(Y, p^{s}\right)$, it implies that $\lim _{n, m \rightarrow \infty} p^{s}\left(x_{n}, x_{m}\right)=0$. Also from (2.3) we have $\lim _{n \rightarrow \infty} p\left(x_{n}, x_{n}\right)=0$, and using the definition of $p^{s}$ we obtain $\lim _{n, m \rightarrow \infty} p\left(x_{n}, x_{m}\right)=0$. Consequently, from (2.4) we have

$$
p(y, y)=\lim _{n \rightarrow \infty} p\left(x_{n}, y\right)=\lim _{n, m \rightarrow \infty} p\left(x_{n}, x_{m}\right)=0 .
$$


As a result, $\left\{x_{n}\right\}$ is a Cauchy sequence in the complete partial metric subspace $(Y, p)$, and it is convergent to a point $y \in Y$.

On the other hand, the sequence $\left\{x_{n}\right\}$ has an infinite number of terms in each $A_{i}, i=$ $1, \ldots, m$. Since $(Y, p)$ is complete, in each $A_{i}, i=1, \ldots, m$, we can construct a subsequence of $\left\{x_{n}\right\}$ which converges to $y$. Since $A_{i}, i=1, \ldots, m$ are closed, we see that

$$
y \in \bigcap_{i=1}^{m} A_{i} ; \text { i.e., }
$$

$\bigcap_{i=1}^{m} A_{i} \neq \emptyset$. Now we can consider the restriction

$$
\left.f\right|_{i=1} ^{m} A_{i}: \quad \bigcap_{i=1}^{m} A_{i} \rightarrow \bigcap_{i=1}^{m} A_{i},
$$

which satisfies the conditions of Theorem 1 in $[5,6]$, since $\bigcap_{i=1}^{m} A_{i}$ is also closed and complete. Thus $\left.f\right|_{i=1} ^{m} A_{i}$ has a unique fixed point, say $x^{*} \in \bigcap_{i=1}^{m} A_{i}$. We claim that for any initial value $x \in Y$, we get the same limit point $x^{*} \in \bigcap_{i=1}^{m} A_{i}$. Indeed, for $x \in Y=\bigcup_{i=1}^{m} A_{i}$, by repeating the above process, the corresponding iterative sequence yields that $\left.f\right|_{i=1} ^{m} A_{i}$ has a unique fixed point, say $z \in \bigcap_{i=1}^{m} A_{i}$. Regarding that $x^{*}, z \in \bigcap_{i=1}^{m} A_{i}$, we have $x^{*} z \in A_{i}$ for all $i$, hence $p\left(x^{*}, z\right)$ and $p\left(f\left(x^{*}\right), f(z)\right)$ are well defined. Due to (2.1), we have

$$
p\left(x^{*}, z\right)=p\left(f\left(x^{*}\right), f(z)\right) \leq \varphi\left(p\left(x^{*}, z\right)\right),
$$

which is a contradiction. Thus, $x^{*}$ is a unique fixed point of $f$ for any initial value $x \in$ $Y$.

To prove that the Picard iteration converges to $x^{*}$ for any initial point $x \in Y$. Let $x \in Y=\bigcup_{i=1}^{m} A_{i}$. There exists $i_{0} \in\{1, \ldots, m\}$ such that $x \in A_{i_{0}}$. As $x^{*} \in \bigcap_{i=1}^{m} A_{i}$ it follows that $x^{*} \in A_{i_{0}+1}$ as well. Then we obtain:

$$
p\left(f(x), f\left(x^{*}\right)\right) \leq \varphi\left(p\left(x, x^{*}\right)\right) .
$$

By induction, it follows that:

$$
p\left(f^{n}(x), x^{*}\right) \leq \varphi^{n}\left(p\left(x, x^{*}\right)\right), \quad n \geq 0 .
$$

Since

$$
p\left(x^{*}, x^{*}\right) \leq p\left(f^{n}(x), x^{*}\right),
$$

we have

$$
p\left(x^{*}, x^{*}\right) \leq \varphi^{n}\left(p\left(x, x^{*}\right)\right) .
$$

Now letting $n \rightarrow \infty$, and supposing $x \neq x^{*}$, we have

$$
p\left(x^{*}, x^{*}\right)=\lim _{n \rightarrow \infty} p\left(f^{n}(x), x^{*}\right)=0,
$$


i.e., the Picard iteration converges to the unique fixed point of $f$ for any initial point $x \in Y$.

Theorem 10 Let $f: Y \rightarrow Y$ as in Theorem 9. Then

$$
\sum_{n=0}^{\infty} p\left(f^{n}(x), f^{n+1}(x)\right)<\infty,
$$

for any $x \in Y$, i.e., $f$ is a good Picard operator.

Proof. Let $x=x_{0} \in Y$. Then

$$
p\left(f^{n}\left(x_{0}\right), f^{n+1}\left(x_{0}\right)\right)=p\left(x_{n}, x_{n+1}\right) \leq \varphi^{n}\left(p\left(x_{0}, x_{1}\right)\right) .
$$

for all $n \in \mathbb{N}$ Thus, by Lemma 6 , we have

$$
\sum_{n=0}^{\infty} p\left(f^{n}\left(x_{0}\right), f^{n+1}\left(x_{0}\right)\right) \leq \sum_{n=0}^{\infty} \varphi^{n}\left(p\left(x_{0}, x_{1}\right)\right)<\infty,
$$

since $p\left(x_{0}, x_{1}\right)>0$. So, $f$ is a good Picard operator.

Theorem 11 Let $f: Y \rightarrow Y$ as in Theorem 9. Then

$$
\sum_{n=0}^{\infty} p\left(f^{n}(x), x^{*}\right)<\infty
$$

for any $x \in Y$, i.e., $f$ is a special Picard operator.

Proof. Since

$$
p\left(f^{n}(x), x^{*}\right) \leq \varphi^{n}\left(p\left(x, x^{*}\right)\right), \quad n \geq 0
$$

holds for any $x \in Y$, by Lemma 6, we have

$$
\sum_{n=0}^{\infty} p\left(f^{n}(x), x^{*}\right) \leq \sum_{n=0}^{\infty} \varphi^{n}\left(p\left(x, x^{*}\right)\right)<\infty .
$$

This shows that $f$ is a special Picard operator.

Theorem 12 (Reich type). Let $(X, p)$ be a complete partial metric space, $m$ a positive integer, $A_{1}, \ldots, A_{m}$ closed nonempty subsets of $X, Y=\bigcup_{i=1}^{m} A_{i}$, and $f: Y \rightarrow Y$ an operator. Assume that

(i) $\bigcup_{i=1}^{m} A_{i}$ is a cyclic representation of $Y$ w.r.t $f$;

(ii) for any $x \in A_{\dot{v}} y \in A_{i+1}$, where $A_{m+1}=A_{1}$, we have

$$
p(f(x), f(y)) \leq \alpha p(x, y)+\beta p(x, f(x))+\gamma p(y, f(y)),
$$

where $\alpha, \beta, \gamma \geq 0$ with $\alpha+\beta+\gamma<1$.

Then $f$ has a unique fixed point $x^{*} \in \bigcap_{i=1}^{m} A_{i}$ and the Picard iteration $\left\{x_{n}\right\}$ converges to $x^{*}$ for any initial point $x_{0} \in Y$ if $\alpha+2 \beta+2 \gamma<1$. 
Proof. Let $x_{0} \in Y=\bigcup_{i=1}^{m} A_{i}$, and set

$$
x_{n}=f\left(x_{n-1}\right), \quad n \geq 1 .
$$

For any $n \geq 0$ there is $i_{n} \in\{i, \ldots, m\}$ such that $x_{n} \in A_{i_{n}}$ and $x_{n+1} \in A_{i_{n+1}}$. Then by (2.5) we have

$$
\begin{aligned}
p\left(x_{n}, x_{n+1}\right) & =p\left(f\left(x_{n-1}\right), f\left(x_{n}\right)\right) \\
& \leq \alpha p\left(x_{n-1}, x_{n}\right)+\beta p\left(x_{n-1}, f\left(x_{n-1}\right)\right)+\gamma p\left(x_{n}, f\left(x_{n}\right)\right) \\
& =\alpha p\left(x_{n-1}, x_{n}\right)+\beta p\left(x_{n-1}, x_{n}\right)+\gamma p\left(x_{n}, x_{n+1}\right) \\
& =(\alpha+\beta) p\left(x_{n-1}, x_{n}\right)+\gamma p\left(x_{n}, x_{n+1}\right),
\end{aligned}
$$

which implies

$$
p\left(x_{n}, x_{n+1}\right) \leq \frac{\alpha+\beta}{1-\gamma} p\left(x_{n-1}, x_{n}\right) .
$$

Therefore,

$$
p\left(x_{n}, x_{n+1}\right) \leq \lambda^{n} p\left(x_{0}, x_{1}\right),
$$

where

$$
\lambda=\frac{\alpha+\beta}{1-\gamma} .
$$

It is clear that $\lambda \in[0,1)$, thus letting $n \rightarrow \infty$ in (2.6), we obtain that

$$
\lim _{n \rightarrow \infty} p\left(x_{n}, x_{n+1}\right)=0 .
$$

On the other hand, since

$$
p\left(x_{n}, x_{n}\right) \leq p\left(x_{n}, x_{n+1}\right) \text { and } p\left(x_{n+1}, x_{n+1}\right) \leq p\left(x_{n}, x_{n+1}\right),
$$

from (2.6) we have

$$
p\left(x_{n}, x_{n}\right) \leq \lambda^{n} p\left(x_{0}, x_{1}\right) \text { and } p\left(x_{n+1}, x_{n+1}\right) \leq \lambda^{n} p\left(x_{0}, x_{1}\right) .
$$

Hence,

$$
p^{s}\left(x_{n}, x_{n+1}\right) \leq 4 \lambda^{n} p\left(x_{0}, x_{1}\right) .
$$

This implies that

$$
\lim _{n \rightarrow \infty} p^{s}\left(x_{n}, x_{n+1}\right)=0 .
$$

Now, for $k \geq 1$, we have

$$
\begin{aligned}
p^{s}\left(x_{n}, x_{n+k}\right) & \leq p^{s}\left(x_{n}, x_{n+1}\right)+\cdots+p^{s}\left(x_{n+k-1}, x_{n+k}\right) \\
& \leq 4 \lambda^{n} p\left(x_{0}, x_{1}\right)+\cdots+4 \lambda^{n+k-1} p\left(x_{0}, x_{1}\right) \\
& \leq 4 \frac{\lambda^{n}}{1-\lambda} p\left(x_{0}, x_{1}\right) .
\end{aligned}
$$

Thus $\left\{x_{n}\right\}$ is a Cauchy sequence in the metric subspace $\left(Y, p^{s}\right)$. Since $Y$ is closed, the subspace $(Y, p)$ is complete and so from Lemma 2, we have that $\left(Y, p^{s}\right)$ is complete. So the sequence $\left\{x_{n}\right\}$ is convergent in the metric subspace $\left(Y, p^{s}\right)$. Let 


$$
\lim _{n \rightarrow \infty} p^{s}\left(x_{n}, y\right)=0
$$

Again from Lemma 2, we get

$$
p(y, y)=\lim _{n \rightarrow \infty} p\left(x_{n}, y\right)=\lim _{n, m \rightarrow \infty} p\left(x_{n}, x_{m}\right) .
$$

As in the proof of Theorem 9, from (2.8) we have

$$
p(y, y)=\lim _{n \rightarrow \infty} p\left(x_{n}, y\right)=\lim _{n, m \rightarrow \infty} p\left(x_{n}, x_{m}\right)=0 .
$$

This shows that $\left\{x_{n}\right\}$ is a Cauchy sequence in the complete partial metric subspace $(Y, p)$, and it is convergent to a point $y \in Y$.

On the other hand, the sequence $\left\{x_{n}\right\}$ has an infinite number of terms in each $A_{i} i=$ $1, \ldots, m$. Since $(Y, p)$ is complete, in each $A_{i}, i=1, \ldots, m$, we can construct a subsequence of $\left\{x_{n}\right\}$ which converges to $y$. Since each $A_{i}, i=1, \ldots, m$ is closed, it follows that

$$
y \in \bigcap_{i=1}^{m} A_{i} ; \text { i.e., }
$$

$\bigcap_{i=1}^{m} A_{i} \neq \emptyset$. Now we can consider the restriction

$$
\left.f\right|_{i=1} ^{m} A_{i}: \bigcap_{i=1}^{m} A_{i} \rightarrow \bigcap_{i=1}^{m} A_{i},
$$

which satisfies the conditions of Corollary 4 in [5], as $\bigcap_{i=1}^{m} A_{i}$ is also closed and com-

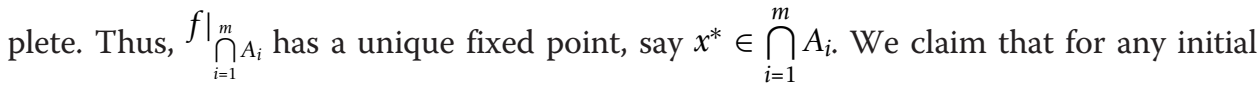
value $x \in Y$, we get the same limit point $x^{*} \in \bigcap_{i=1}^{m} A_{i}$. In fact, for $x \in Y=\bigcup_{i=1}^{m} A_{i}$, by repeating the above process, the corresponding iterative sequence yields that $\left.f\right|_{i=1} ^{m} A_{i}$ has a unique fixed point, say $z \in \bigcap_{i=1}^{m} A_{i}$. Since $x^{*}, z \in \bigcap_{i=1}^{m} A_{i}$, we have $x^{*}, z \in A_{i}$ for all $i$, hence $p\left(x^{*}, z\right)$, and $p\left(f\left(x^{*}\right), f(z)\right)$ are well defined. Due to (2.5),

$$
\begin{aligned}
p\left(x^{*}, z\right) & =p\left(f\left(x^{*}\right), f(z)\right) \\
& \leq \alpha p\left(x^{*}, z\right)+\beta p\left(x^{*}, f\left(x^{*}\right)\right)+\gamma p(z, f(z)) \\
& \leq \alpha p\left(x^{*}, z\right)+\beta p\left(x^{*}, z\right)+\gamma p\left(x^{*}, z\right),
\end{aligned}
$$

which is a contradiction. Thus, $x *$ is the unique fixed point of $f$ for any initial value $x$ $\in Y$.

To prove that the Picard iteration converges to $x^{*}$ for any initial point $x \in Y$. Let $x \in Y=\bigcup_{i=1}^{m} A_{i}$. There exists $i_{0} \in\{1, \ldots, m\}$ such that $x \in A_{i_{0}}$. As $x^{*} \in \bigcap_{i=1}^{m} A_{i}$ it follows that $x^{*} \in A_{i_{0}+1}$ as well. Then we obtain:

$$
\begin{aligned}
p\left(f(x), f\left(x^{*}\right)\right) \leq & \alpha p\left(x, x^{*}\right)+\beta p(x, f(x))+\gamma p\left(x^{*}, f\left(x^{*}\right)\right) \\
\leq & \alpha p\left(x, x^{*}\right)+\beta\left[p\left(x, x^{*}\right)+p\left(x^{*}, f(x)\right)-p\left(x^{*}, x^{*}\right)\right] \\
& +\gamma\left[p\left(x^{*}, f(x)\right)+p\left(f(x), f\left(x^{*}\right)\right)-p(f(x), f(x))\right] \\
\leq & \alpha p\left(x, x^{*}\right)+\beta\left[p\left(x, x^{*}\right)+p\left(x^{*}, f(x)\right)\right] \\
& +\gamma\left[p\left(x^{*}, f(x)\right)+p\left(f(x), f\left(x^{*}\right)\right)\right],
\end{aligned}
$$


which implies

$$
p\left(f(x), f\left(x^{*}\right)\right) \leq \frac{\alpha+\beta}{1-\beta-2 \gamma} p\left(x, x^{*}\right) .
$$

Let

$$
\lambda_{1}=\frac{\alpha+\beta}{1-\beta-2 \gamma},
$$

and suppose that $\alpha+2 \beta+2 \gamma<1$. Then, by induction, it follows that:

$$
p\left(f^{n}(x), x^{*}\right) \leq \lambda_{1}^{n} p\left(x, x^{*}\right) .
$$

Since

$$
p\left(x^{*}, x^{*}\right) \leq p\left(f^{n}(x), x^{*}\right),
$$

we have

$$
p\left(x^{*}, x^{*}\right) \leq \lambda_{1}^{n} p\left(x, x^{*}\right) .
$$

Now letting $n \rightarrow \infty$, and supposing $x \neq x^{*}$, we have

$$
p\left(x^{*}, x^{*}\right)=\lim _{n \rightarrow \infty} p\left(f^{n}(x), x^{*}\right)=0
$$

i.e., the Picard iteration converges to the unique fixed point of $f$ for any initial point $x \in Y$ provided $\alpha+2 \beta+2 \gamma<1$.

Corollary 13 (Banach type). Let $(X, p)$ be a complete partial metric space, $m$ a positive integer, $A_{1}, \ldots, A_{m}$ closed nonempty subsets of $X, Y=\bigcup_{i=1}^{m} A_{i}$, and $f: Y \rightarrow Y$ an operator. Assume that

(i) $\bigcup_{i=1}^{m} A_{i}$ is a cyclic representation of $Y$ w.r.t $f$;

(ii) for any $x \in A_{\dot{v}} y \in A_{i+1}$, where $A_{m+1}=A_{1}$, we have

$$
p(f(x), f(y)) \leq \alpha p(x, y), \quad 0 \leq \alpha<1 .
$$

Then $f$ has a unique fixed point $x^{*} \in \bigcap_{i=1}^{m} A_{i}$.

Corollary 14 (Kannan type). Let $(X, p)$ be a complete partial metric space, $m$ a positive integer, $A_{1}, \ldots, A_{m}$ closed nonempty subsets of $X, Y=\bigcup_{i=1}^{m} A_{i}$, and $f: Y \rightarrow Y$ an operator. Assume that

(i) $\bigcup_{i=1}^{m} A_{i}$ is a cyclic representation of $Y$ w.r.t $f$;

(ii) for any $x \in A_{\dot{v}} y \in A_{i+1}$, where $A_{m+1}=A_{1}$, we have

$$
p(f(x), f(y)) \leq \beta p(x, f(x))+\gamma p(y, f(y)),
$$

where $\beta, \gamma \geq 0$ with $\beta+\gamma<\frac{1}{2}$.

Then $f$ has a unique fixed point $x^{*} \in \bigcap_{i=1}^{m} A_{i}$. 
Theorem 15 Let $f: Y \rightarrow Y$ as in Theorem 12. Then

$$
\sum_{n=0}^{\infty} p\left(f^{n}(x), f^{n+1}(x)\right)<\infty
$$

for any $x \in Y$, i.e., $f$ is a good Picard operator.

Proof. Let $x=x_{0} \in Y$. Then, as in the proof of Theorem 12,

$$
p\left(f^{n}\left(x_{0}\right), f^{n+1}\left(x_{0}\right)\right)=p\left(x_{n}, x_{n+1}\right) \leq \lambda^{n} p\left(x_{0}, x_{1}\right)
$$

for all $n \in \mathbb{N}$. So, we have

$$
\sum_{n=0}^{\infty} p\left(f^{n}\left(x_{0}\right), f^{n+1}\left(x_{0}\right)\right) \leq \sum_{n=0}^{\infty} \lambda^{n} p\left(x_{0}, x_{1}\right)<\infty,
$$

since $\lambda \in[0,1)$. Thus, $f$ is a good Picard operator.

Theorem 16 Let $f: Y \rightarrow Y$ as in Theorem 12. If $\alpha+2 \beta+2 \gamma<1$, then

$$
\sum_{n=0}^{\infty} p\left(f^{n}(x), x^{*}\right)<\infty
$$

for any $x \in Y$, i.e., $f$ is a special Picard operator.

Proof. As in the proof of Theorem 12, we have

$$
p\left(f^{n}(x), x^{*}\right) \leq \lambda_{1}^{n} p\left(x, x^{*}\right)
$$

holds for any $x \in Y$, where $\lambda_{1}=\frac{\alpha+\beta}{1-\beta-2 \gamma}$. Hence, if $\alpha+2 \beta+2 \gamma<1$, we have

$$
\sum_{n=0}^{\infty} p\left(f^{n}(x), x^{*}\right) \leq \sum_{n=0}^{\infty} \lambda_{1}^{n} p\left(x, x^{*}\right)<\infty .
$$

This shows that $f$ is a special Picard operator.

\section{Acknowledgements}

The second and third authors would like to thank the Deanship of Scientific Research (DSR) at the King Abdulaziz University, Jeddah for supporting this work through research project No. 1432/363/31.

\section{Author details}

${ }^{1}$ Department of Mathematics, Texas A\&M University-Kingsville 700 University Blvd. Kingsville, TX 78363-8202, USA

${ }^{2}$ Department of Mathematics, King Abdulaziz University, Sciences Faculty for Girls, P.O. Box 4087, Jeddah 21491, Saudi Arabia ${ }^{3}$ Department of Mathematics, King Abdulaziz University, P.O. Box 80203, Jeddah 21859, Saudi Arabia

\section{Authors' contributions}

All authors contributed equally and significantly in writing this paper. All authors read and approved the final manuscript.

\section{Competing interests}

The authors declare that they have no competing interests.

Received: 16 September 2011 Accepted: 15 March 2012 Published: 15 March 2012

\section{References}

1. Kannan, R: Some results on fixed points. Bull Calcutta Math Soc. 60, 71-76 (1968)

2. Reich, S: Kannan's fixed point theorem. Boll Unione Mat Ital. 4(4):1-11 (1971)

3. Matkowski, J: Fixed point theorems for mappings with a contractive iterate at a point. Proc Amer Math Soc. 62(2):344-348 (1977). doi:10.1090/S0002-9939-1977-0436113-5

4. Matthews, SG: Partial metric topology. Papers on General Topology and Applications (Flushing, NY, 1992). Ann New York Acad Sci. 728, 183-197 (1994). doi:10.1111/j.1749-6632.1994.tb44144.x

5. Altun, I, Sola, F, Simsek, H: Generalized contractions on partial metric spaces. Topol Appl. 157, 2778-2785 (2010). doi:10.1016/j.topol.2010.08.017 
6. Altun, I, Sadarangani, K: Corrigendum to generalized contractions on partial metric spaces. Topol Appl 158, 1738-1740 (2011). [Topol. Appl. 157, 2778-2785 (2010)]. doi:10.1016/j.topol.2011.05.023

7. Di Bari, C, Vetro, P: Fixed points results for weak $\varphi$ contractions on partial metric spaces. Int J Eng Contemp Math Sci (2011). (to appear)

8. Valero, O: On Banach fixed point theorems for partial metric spaces. Appl Gen Topol. 6(2):229-240 (2005)

9. Ciric, Lj, Samet, B, Aydi, H, Vetro, C: Common fixed points of generalized contractions on partial metric spaces and an application. Appl Math Comput. 218, 2398-2406 (2011). doi:10.1016/j.amc.2011.07.005

10. Escardo, MH: Pcf extended with real numbers. Theor Comput Sci. 162, 79-115 (1996). doi:10.1016/0304-3975(95)00250-2

11. Schellekens, M: The smyth completion: a common foundation for denonational semantics and complexity analysis. Proc Mathematical foundations of programming semantics (New Orleans, LA 1995), Electronic Notes in Theoretical Computer Science. 1, 211-232 (1995)

12. Oltra, S, Valero, O: Banach's fixed point theorem for partial metric spaces. Rend Istit Mat Univ Trieste. 36, 17-26 (2004)

13. Păcurar, M, Rus, IA: Fixed point theory for cyclic $\varphi$-contractions. Nonlinear Anal. 72(4-3):1181-1187 (2010)

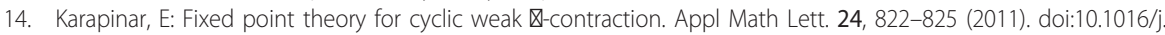
aml.2010.12.016

doi:10.1186/1687-1812-2012-40

Cite this article as: Agarwal et al:: Fixed point theory for cyclic generalized contractions in partial metric spaces. Fixed Point Theory and Applications 2012 2012:40.

\section{Submit your manuscript to a SpringerOpen ${ }^{\circ}$} journal and benefit from:

- Convenient online submission

- Rigorous peer review

- Immediate publication on acceptance

- Open access: articles freely available online

- High visibility within the field

- Retaining the copyright to your article

Submit your next manuscript at $\gg$ springeropen.com 